Manuelle Medizin 2020 · 58:204-209

https://doi.org/10.1007/s00337-020-00702-5

Online publiziert: 2 . Juli 2020

(c) Der/die Autor(en) 2020

Der Beckenboden (BB) ist in seinem strukturellen Aufbau und seiner Funktion ein komplexer Körperbereich. Er erfährt im Alterungsprozess physiologische Veränderungen, ist in den Lebensphasen aber auch geschlechterspezifisch unterschiedlich belastenden Prozessen ausgesetzt. Nichtoperative Behandlungsansätze am BB fokussieren auf das Training der Muskelfunktion. Zu diskutieren ist, ob Interventionen, die das fasziale Gewebe des BB und dessen propriozeptive Funktion ansprechen, gerade im Alter mehr Beachtung finden sollten.

Der Beckenboden (BB) bildet die kaudale Begrenzung des Becken-BauchRaums. Er überspannt die Innenseite des Beckenrings, ist mehrschichtig angelegt und multidimensional ausgerichtet ([1]; - Abb. 1). Interessant ist der hohe fasziale Anteil innerhalb der BB-Strukturen. Der BB erfüllt mehrere sehr unterschiedliche Funktionen. Er stützt die Beckenorgane Blase und Enddarm von kaudal ab und sichert deren topographische Lage während der ständig ablaufenden Füllungs- und Entleerungsvorgänge. Zudem sichert er wichtige Lage- und Winkelverhältnisse am vesikourethralen und anorektalen Übergang und unterstützt so die Speicherung und Entleerung von Harn und Stuhl (• Abb. 2). In dieser Funktion besteht ein im menschlichen Körpersystem einzigartiges Zusammenspiel zwischen den vegetativ unwillkürlich gesteuerten Ausscheidungsorganen und dem somatisch willkürlich innervierten BBSystem. Bei der Frau stabilisiert der BB das untere Drittel der Scheide und

Barbara Gödl-Purrer

Physiotherapie, FH JOANNEUM, Graz, Österreich

\title{
Der Beckenboden im Alter
}

\section{Überlegungen zu einem funktionellen Behandlungsansatz}

indirekt die Gebärmutter. Beim Mann werden der Blasenfundus und das obere Drittel der Urethra über die Prostatadrüse mechanisch gestützt. Dies entlastet relativ die Supportfunktion des BB. Bei operativen Eingriffen an der Prostata wird dieses mechanisch stabile System wesentlich labilisiert.

Der BB steht in einem synergistischen Zusammenspiel mit der „Rumpfkapsel“ $[2,3]$. In diesem System werden über die ständig ablaufenden Atembewegungen des Diaphragma pulmonale intraabdominelle Druckwechsel erzeugt, die an der Bauch- und Rückenwand sowie dem BB Tonusanpassungen im Sinne eines exzentrischen Nachgebens und konzentrischen „Rückfederns“ fordern [4]. Somit wird die Stabilisationsleistung dieser Rumpfsysteme zu einer dynamischen Funktion, was für die Stoffwechselprozesse in den Geweben und die Erhaltung einer hohen Reaktionsbereitschaft als sinnvoll erachtet werden kann.

Als innerer Stabilisator des Beckenrings überspannt der BB das iliosakrale Gelenk und die Symphyse. Durch seine ventrodorsale Ausrichtung zwischen Os coccygis und Sakrum nimmt er Einfluss auf die Nutationsstellung des Sakrums und die Extensionsstellung im lumbosakralen Übergang [6]. Über die gemeinsame Ansatzstelle des M. obturatorius internus und M. levator ani steht der $\mathrm{BB}$ in einer Spannungsübertragung aus der unteren Extremität und reagiert auf Beinbewegungen $[5,6]$.

\section{Beckenboden und Alter}

Die Strukturen des BB unterliegen physiologischen Alterungsprozessen. Auf muskulärer Ebene hat dies ab dem
60. Lebensjahr eine Abnahme von Muskelmasse zur Folge [7]. Zusätzlich kommt es im Bindegewebe zu einer Reduktion von Elastizität, Hydratation und Gleitfähigkeit. Dies führt zu einer verminderten Fähigkeit, die Funktionen des $\mathrm{BB}$ sicher und zuverlässig zu erfüllen, und erklärt die in der Literatur beschriebenen Dysfunktionen der Miktion und Defäkation sowie Zustände von Organsenkung. Trowbridge et al. [8] konnten bei 82 Nullipara im Alter von 21 bis 70 Jahren feststellen, dass mit höherem Alter durch Reduktion der willkürlichen Muskelfasern und der Faserdichte im M. sphincter urethrae externus der maximale urethrale Verschlussdruck um $15 \mathrm{~cm} \mathrm{H}_{2} \mathrm{O}$ pro Dekade sinkt. Zudem führen Umbauprozesse des paraurethralen Gewebes dazu, dass die bindegewebigen Anteile höher werden. Die Durchblutung vermindert sich und es kommt zu einer reduzierten nervalen Versorgung. Burnett et al. [9] fanden in einer Kadaverstudie eine altersabhängige Zunahme der Gewebesteifigkeit im weiblichen $\mathrm{BB}$ und eine Abnahme von Belastbarkeit, Dehnbarkeit und Kontraktilität betroffener myofaszialer Strukturen. Der Verlust an Elastizität und der Abbau von faszialem Tonus wird als Ursache für eine verminderte Rekrutierungsfähigkeit und muskulärer Kraft diskutiert [10]. Verändert sich zudem die propriozeptive Innervation im Gewebe, wird sich dies auf die Repräsentation des BB im Körperbild und die selektive Körperwahrnehmung auswirken [11]. DeLancey et al. [12] geben an, dass $11 \%$ aller Frauen eine derart starke Form der BB-Dysfunktion erleben, dass ein operativer Eingriff notwendig wird. 


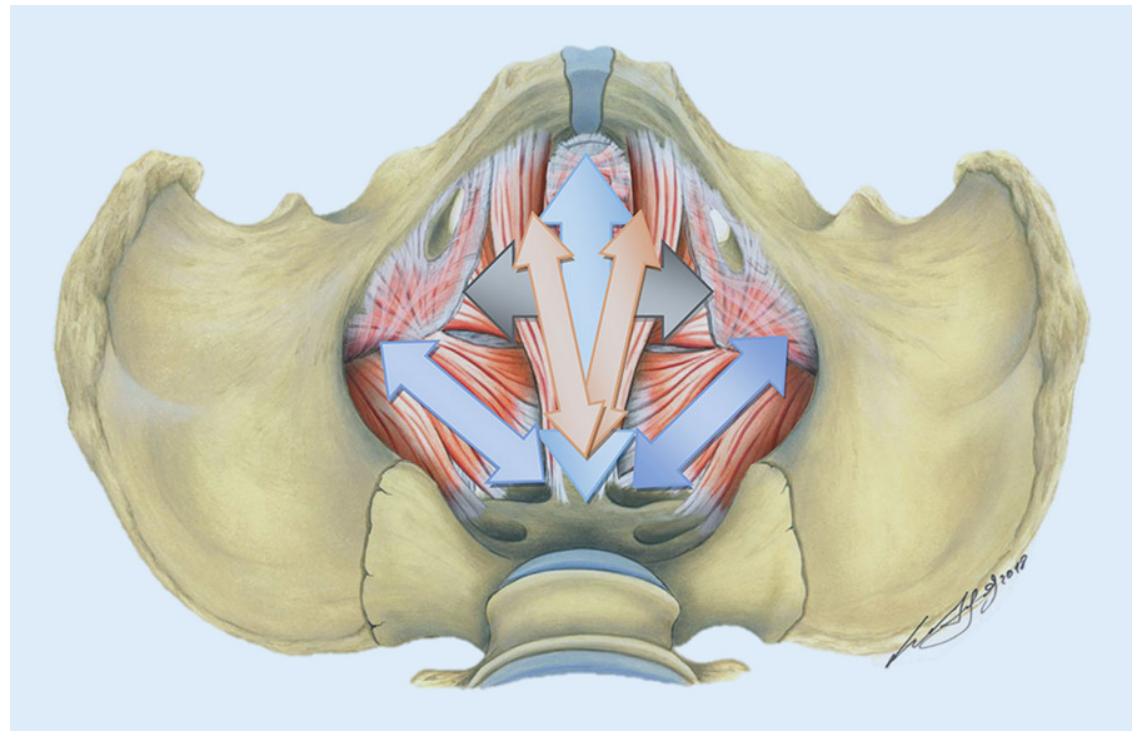

Abb. $1 \Delta$ Der Beckenboden wirkt als Stabilisator der Beckenorgane und des Beckenrings. (Mit freundl. Genehmigung, @ Andreas Bauer, Lehrstuhl für makroskopische und klinische Anatomie, Medizinische Universität Graz, Zeichnungen, alle Rechte vorbehalten)

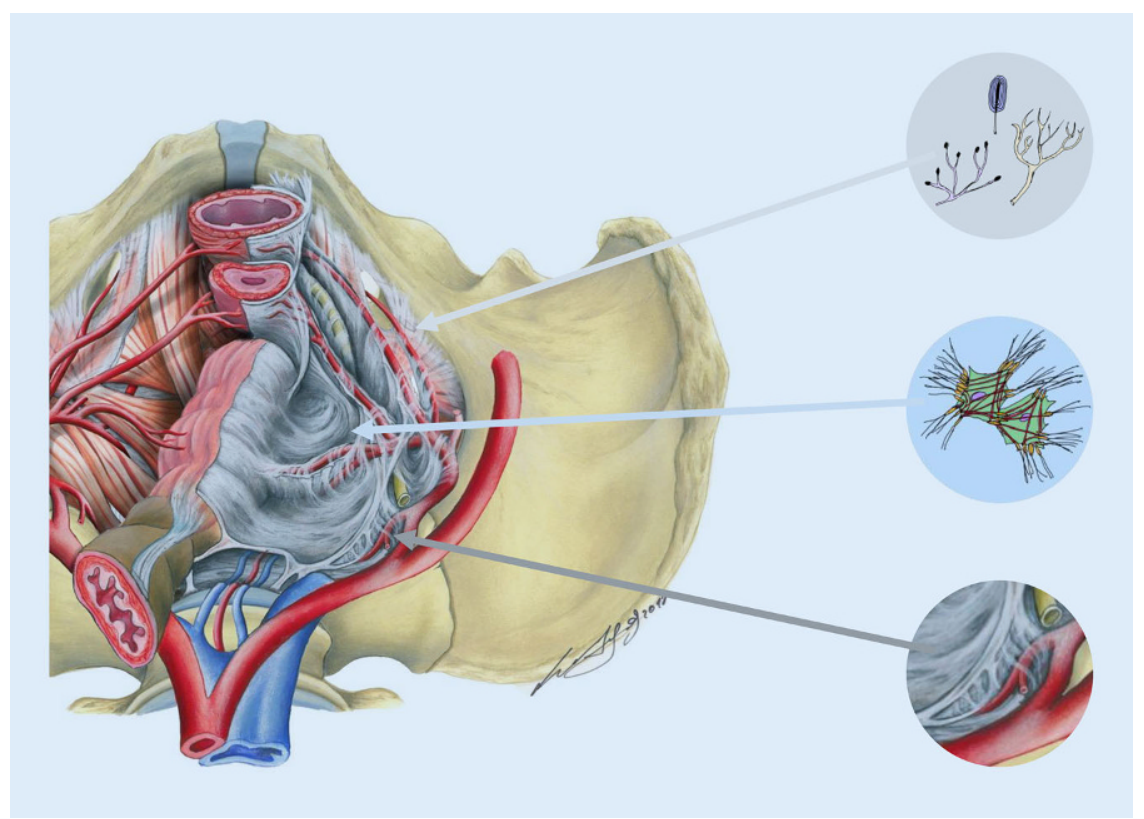

Abb. $2 \triangle$ Mechanosensoren beeinflussen die sensorische Repräsentation des Beckenbodens (BB) im Gehirn. Kollagene Fasern organisieren sich bezüglich Ausrichtung, Dichte und Elastizität durch mechanische Reize. Die Gleitfähigkeit der Strukturen des BB sorgt für deren ungestörte Funktion. (Mit freundl. Genehmigung, $\odot$ Andreas Bauer und Dr. Andreas Sammer, Lehrstuhl für makroskopische und klinische Anatomie, Medizinische Universität Graz, Zeichnungen, alle Rechte vorbehalten)

Die Häufigkeit aller Formen der Harninkontinenz steigt bei Frauen nach der Menopause deutlich an und wird mit einer Inzidenz von $20-40 \%$ bei älteren Frauen beschrieben [13]. Harninkontinenz ist neben Demenz und Stuhlinkontinenz bei den über 65-Jährigen eine der häufigsten Alterserkrankungen. Bei

\section{Beckenboden im Lebenszeitmodell}

DeLancey et al. [12] beschreiben für das Altern des weiblichen BB ein 3-phasiges „life span model“ und suchen nach Erklärungsmodellen für Faktoren und Interdependenzen, die den Alterungsprozess beeinflussen. Für den männlichen $\mathrm{BB}$ gibt es kein vergleichbares Modell, jedoch können die Ansätze der Lebensphasen für beide Geschlechter betrachtet werden. Als „prädisponierende“ Phase beschreiben die Autoren die Zeit des Wachstums und der Entwicklung. In dieser Zeit werden über genetisch determinierende und Lebensstilfaktoren Ressourcen aufgebaut, die den Grundstein für eine gute Resistenz gegen spätere negativ beeinflussende Faktoren bilden. In einer 2. Phase wirken Belastungen wie z.B. Schwangerschaften und Geburten oder operative Eingriffe an den Beckenorganen auf das BB-System. Ohne adäquate Behandlung bleiben Narben, Verlust an Elastizität und Gleitfähigkeit sowie reduzierte Mechanosensitivität als Quellen für Funktionsdefizite bestehen und stören die BB-Funktion möglicherweise nachhaltig. Die 3. Lebenszeitphase ist gekennzeichnet durch den fortschreitenden Alterungsprozess. Neben den degenerativen Veränderungen in Muskel- und Bindegewebe kommt es zur Reduktion des allgemeinen Leistungszustands. Zusätzlich können Krankheiten des metabolischen, kardiorespiratorischen, neurogenen und psychischen Systems zum Auftreten von BB-Dysfunktionen beitragen. Dies kann dazu führen, dass sich die Belastungen aus der 2. Phase nicht mehr kompensieren lassen.

\section{Behandlungsansatz „Muskel- training "}

Die Wirksamkeit eines selektiven Beckenbodentrainings (BBT) auf Heilung oder Symptomminderung bei der weiblichen Harninkontinenz und Organsenkung ist wissenschaftlich sehr gut nachgewiesen [15]. Daher besteht eine evidenzbasierte Empfehlung für das BBT als First-Line-Behandlungsansatz bei Harninkontinenz [16]. Keine eindeutige Aussage gibt es zu Best-PracticeModellen für BBT [17]. Jedoch scheint
Männern treten Kontinenzstörung später auf und werden meist primär durch operative Eingriffe an der Prostata ausgelöst. Die Prävalenz für alle Formen der männlichen Kontinenzstörung liegt bei $4,4 \%$ [14]. 
es erwiesen $\mathrm{zu}$ sein, dass am Beginn jedes Trainings die Instruktion der korrekten isolierten Rekrutierungsfähigkeit der BB-Muskulatur stehen soll [16].

Für die Wirksamkeit des BBT auf die männliche Harninkontinenz gibt es bis dato keine eindeutigen wissenschaftliche Nachweise [18]. Allerdings stellt sich die Frage, ob die Trainingsansätze, die bei Frauen wirksam sind, auf den Mann übertragen werden können. So beschreiben Stafford et al. [19] ein Modell der männlichen Kontinenzmechanismen, das als Zielmuskulatur beim Mann das ventrale BB-Kompartment in den Vordergrund rückt.

\section{》) Gezieltes und intensives Muskeltraining kann bei Älteren wirksam eingesetzt werden}

Nachgewiesen wurde, dass ein gezieltes und intensives Muskeltraining bei Älteren wirksam eingesetzt werden kann [20, 21]. Talasz et al. [22] untersuchten 377 geriatrische Patientinnen mit Harninkontinenz auf die korrekte willkürliche Rekrutierungsfähigkeit der BB-Muskulatur. Etwa $65,5 \%$ der Patientinnen hatten keine, $22 \%$ eine insuffiziente und nur $12,5 \%$ eine sichere Muskelkontraktionsfähigkeit. Von den Frauen, die bereits ein BBT absolviert hatten, konnten nur solche korrekt und kraftvoll den BB anspannen, die über digitale Feedbackverfahren individuell instruiert worden waren.

Besonders bei älteren Personen ist es daher wichtig, die selektive Rekrutierungsfähigkeit für die BB-Muskulatur zu schulen [22]. Taktile und gerätegestützte Feedbackmethoden oder die Verwendung von zusätzlichen Innervationshilfen wie funktionelle Elektrostimulation können gut eingesetzt werden und ermöglichen das Erlernen der korrekten Anspannung als Voraussetzung für ein Training in allen Altersstufen (• Tab. 1). Formen des Hypertrophietrainings tragen zum Erhalt von Muskelmasse und Faserdichte bei. Ein Training der Schnellkraft und des „pre-timing“, also einer zeitlich vorgeschalteten BB-Kontraktion bei schnellen intraabdominellen Druckerhöhungen, wirkt auf die motorische Kontrollfähigkeit [23].

Manuelle Medizin 2020 · 58:204-209 https://doi.org/10.1007/s00337-020-00702-5

(c) Der/die Autor(en) 2020

\section{B. Gödl-Purrer}

\section{Der Beckenboden im Alter. Überlegungen zu einem funktionellen Behandlungsansatz}

\section{Zusammenfassung}

Der Beckenboden (BB) ist eine komplexe Struktur. Er erfüllt multiple Funktionen, von denen die Lagesicherung der Beckenorgane und der damit verbundene Einfluss auf die Harn- und Stuhlkontinenz bereits gut erforscht sind. Das BB-Gewebe wird durch die physiologischen Alterungsprozesse wesentlich geschwächt. Zusätzlich belasten Lebensevents wie Schwangerschaft und Geburt, operative Eingriffe, aber auch typische Alterserkrankungen wie Diabetes, Lungen- und Stoffwechselerkrankungen diesen Körperbereich. Die Wirksamkeit des Trainings der BB-Muskulatur auf die Kontinenz und Organsenkung ist wissenschaftlich auch in der älteren Bevölkerung bewiesen. Therapeutische Konzepte, die auf die Behandlung des Fasziengewebes im BB fokussieren, werden aktuell nicht standardisiert eingesetzt. Da Alterungsprozesse zu Bindegewebeveränderungen führen, erscheint der Einsatz von Faszientechniken jedoch überlegenswert.

Schlüsselwörter

Altern · Harninkontinenz · Körperliche Aktivität · Muskuloskeletales System · Faszie

\section{The pelvic floor in the elderly. Considerations for a functional treatment approach}

\section{Abstract}

The pelvic floor (PF) is a complex structure. Its functions are multifold, but the PF as a support system for the pelvic organs and its influence on urinary and fecal continence are those best researched. The physiologic aging process significantly weakens the myofascial tissue of the PF. In addition, events such as pregnancy and childbirth, surgery, and common diseases of older age such as diabetes, lung diseases, and metabolic syndromes put additional strain on the PF. Research has shown that training of the PF muscles is also effective in the rehabilitation of incontinence conditions and pelvic organ prolapse in elderly persons. Therapeutic techniques focused on treatment of PF fascial tissue are not currently applied in a standardized manner. However, as aging processes change connective tissue, the use of fascial techniques would appear to represent a promising approach.

Keywords

Aging · Urinary incontinence - Physical activity · Musculoskeletal system · Fascia

\section{Propriozeption und senso- motorische Kontrolle}

Im BB finden sich unterschiedliche Formen von „faszialem“ Gewebe. Forschungsergebnisse zeigen, dass Faszien propriozeptiv innerviert sind und daher als Wahrnehmungsorgan eine hohe Bedeutung in der sensomotorischen Kontrollfunktion haben ([10, 24]; • Abb. 2). Für den Aufbau einer belastbaren Faserarchitektur und für die physiologische Organisation kollagener Fasernetzwerke wird eine mechanisch gerichtete funktionelle Nutzung benötigt. Propriozeptiv afferente Informationen sind für das sensorische Feedback von Bewegungs- funktionen in Haltung und Bewegung zuständig. Sie sind wichtig, um die muskuläre Feinabstimmung in der Bewegungsausführung $\mathrm{zu}$ gewährleisten. Diese Funktion läuft unwillkürlich und unbewusst ab. Häufig gebrauchte alltägliche Bewegungsmuster werden als automatisiertes sensomotorisches Muster im Gehirn gespeichert [11]. Durch Üben und gezielte Wahrnehmungslenkung können kinästhetische Sinnesqualitäten bewusst gemacht werden. Den Übenden wird damit eine Möglichkeit gegeben, Körperbereiche selektiv zu spüren und differenziert zu bewegen. 


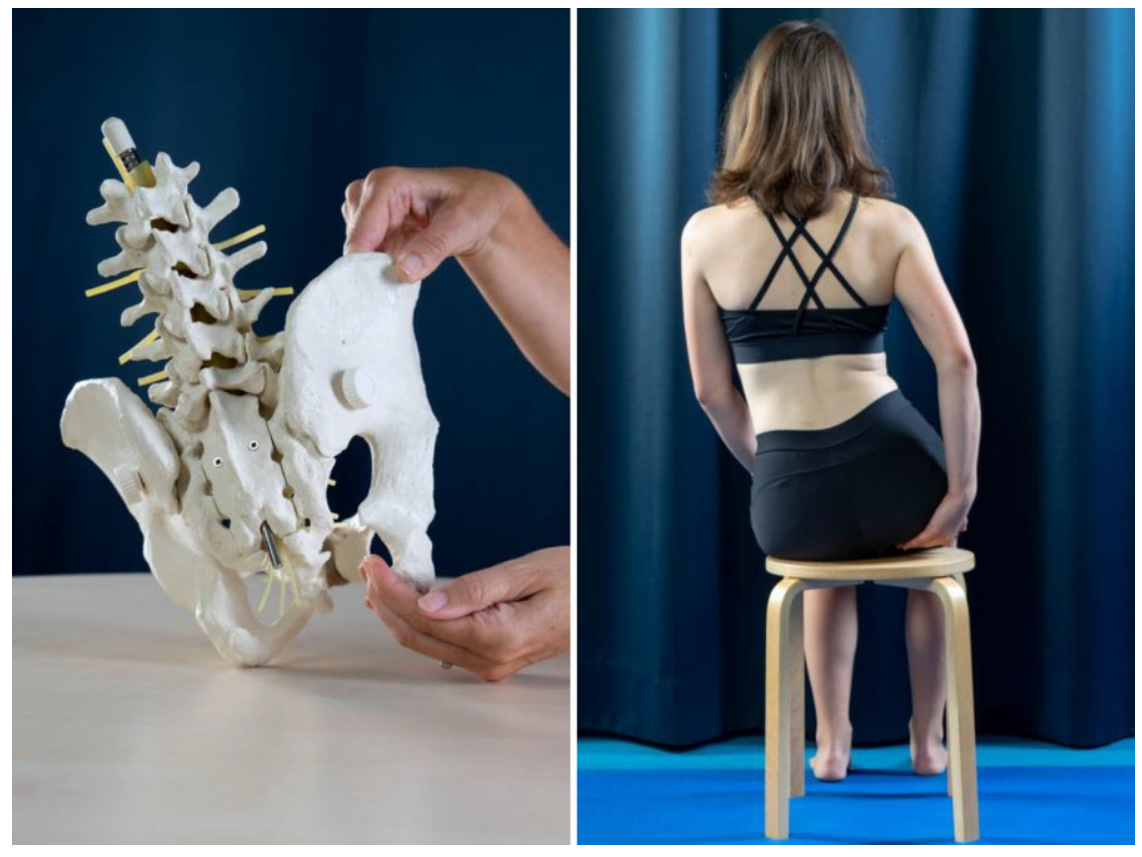

Abb. 3 A Durch manuelle Palpation werden Wahrnehmung und Orientierung am knöchernen Becken angelegt, in der Eigenübung umgesetzt und im Alltag genutzt

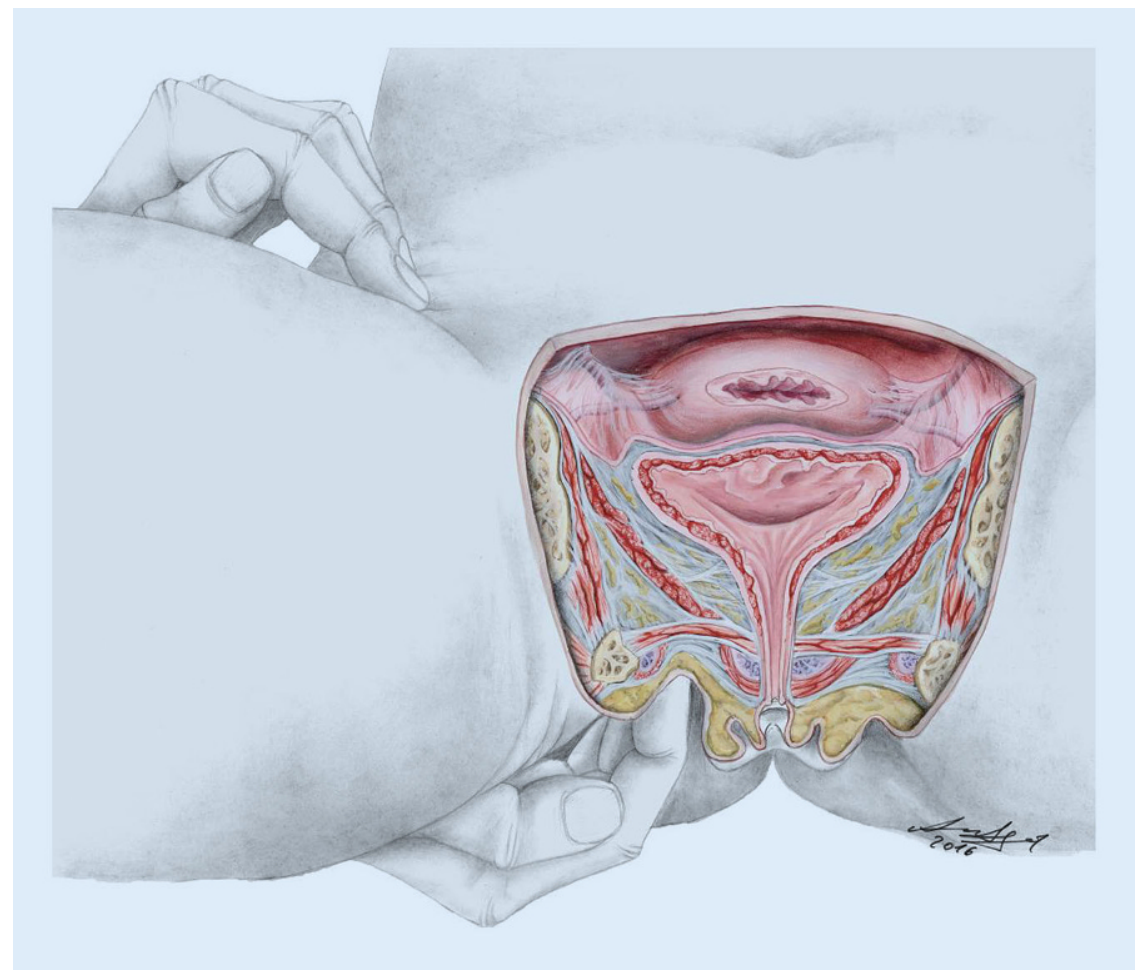

Abb. 4 A Einwirkung auf die Beckenbodenstrukturen durch manuelle Mobilisationen. (Aus Sammer [25], mit freundl. Genehmigung $\odot$ Andreas Bauer und Dr. Andreas Sammer, Lehrstuhl für makroskopische und klinische Anatomie, Medizinische Universität Graz, alle Rechte vorbehalten)

\section{Behandlungsansatz „proprio- zeptive Integration"}

Faszientechniken arbeiten über manuell gesetzte Gewebereize und werden zur Behandlung dysfunktionaler Zustände am Bindegewebe (z. B. fasziale Steifigkeit, Narben, Triggerpunkte) eingesetzt. Diese manuellen Techniken sind geeignet, um die Mechanosensitivität im Gewebe zu beeinflussen und dadurch in die sensomotorische Steuerung einzugreifen. Gekoppelt mit Wahrnehmungsübungen kann somit auf die Qualität der motorischen Kontrollfunktion eingewirkt werden. Aktuell werden derartige Behandlungsansätze am BB nicht standardisiert durchgeführt und die Wirksamkeit ist klinisch nicht erforscht. Die nachfolgend dargestellten 3 Prinzipien eines Behandlungsansatzes mit Fokus auf die Beeinflussung der faszialen Funktion wurden an v.a. älteren Patienten erprobt. Erste Erfahrungen zeigen gute Behandlungsergebnisse und eine hohe Patientenakzeptanz.

\section{Prinzip 1: Wahrnehmung und Selektivität}

Ziel der Interventionen ist es, über die Aktivierung von Mechanosensoren ein differenziertes Körperbild des Beckens und des BB anzulegen oder zu erhalten. Durch Palpation am knöchernen Becken und an den zentralen faszialen Gewebestrukturen wird die Wahrnehmung gelenkt. Patienten werden zum bewussten „Spüren" geführt, indem sie Lokalisation, Stärke, Distanzen und Bewegungsrichtungen der Kontakte differenziert erleben und bewusst rückmelden ( $\mathbf{0}$ Abb. 3 ). Der Effektnachweis kann über Tests der Oberflächen- und Tiefensensibilität erfolgen.

\section{Prinzip 2: innere und äußere Gleitfähigkeit}

Der Stoffwechsel soll lokal angeregt, die intrafasziale Mobilität verbessert und die selektive Rekrutierung von Muskeln stimuliert werden. Durch richtunggebende Reize in geringer bis gesteigerter Druckintensität werden Informationen entsprechend der myofaszialen Gewe- 


\section{Tab. 1 Korrekte Anspannung des Beckenbodens (BB)}

Bei der Frau Die BB-Anspannung führt zur Verengung von Vagina, Urethra und Analkanal und zu einer Elevation des Blasenhalses sowie des anorektalen Übergangs Kontrolle über Palpation: intravaginal, intraanal oder medial der Tuber ossis ischii

Kontrolle über Inspektion: am Perineum bzw. Introitus vaginae, Anus; Bildgebend über perinealen Echtzeitultraschall

Beim Mann Die BB-Anspannung führt zur Verengung von Urethra und Analkanal und zu einer Elevation des Blasenhalses und des anorektalen Übergangsbereichs

Kontrolle über Palpation: intraanal oder extern am Bulbus penis und medial der Tuber ossis ischii

Kontrolle über Inspektion: am Perineum, Anus, Bulbus penis; Bildgebend über perinealen Echtzeitultraschall

Reaktive Aktivierung des BB: Beim Räuspern, verlängerten Ausatmen und Sprechen kommt es zu einer tastbaren und/oder sichtbaren reaktiven Elevation des Blasenhalses sowie des anorektalen Übergangs oder am Perineum

beausrichtung und der Kontraktionsrichtung von BB-Muskulatur in das Bindegewebe gegeben. Durch bewusste Wahrnehmungslenkung erfasst der Patient die Differenziertheit und Selektivität der „Bewegungsmöglichkeiten“ im BB (•Abb. 4). Über tiefe, langsam ausgeführte Drucktechniken werden die Hydratation der Gewebe sowie ein Lösen von Querverbindungen zwischen Gewebeschichten und an Gleitflächen zwischen Muskelschichten und inneren Organen angestrebt. Der fasziale Tonus wird beeinflusst [10]. Bekannte Faszientechniken, z.B. „fascial release“, Triggerpunktbehandlung und viszerale Techniken, können zum Einsatz kommen. Der Effekt lässt sich über Tests der Muskelrekrutierung und vergleichende Palpation der BB-Gewebe nachweisen.

\section{Prinzip 3: Integration und Koordination}

Interventionen üben die BB-Funktion integriert in das myofasziale Kettensystem und in synergistische Funktionsketten des Bewegungssystems. Aktivitäten, die den BB in physiologischer Weise nutzen, werden angeleitet. Die Aktivierung des $\mathrm{BB}$ erfolgt als reaktives Geschehen in der motorischen Kontrolle von Alltagsbewegungen. Besonders geeignet sind Aktivitäten, die die Atmung nutzen bzw. die Atemfrequenz und -tiefe variieren wie das Sprechen oder Singen, aber auch Gehbewegungen und gymnastische Übungen, die die BB-Muskulatur in der Kettenfunktion ansteuern. Der Effektnachweis kann über Symptomkontrolle in Kennbewegungen und über vergleichende Palpation erfolgen.

\section{Fazit für die Praxis}

- Die physiologischen Veränderungen am BB-Gewebe führen beim alten Menschen zu einer Fülle von Funktionsstörungen; die Harn- und Stuhlinkontinenz ist die am meisten untersuchte Form.

- Die Symptome der Kontinenzstörung belasten das soziale Leben alter Menschen wesentlich, was die soziale Vereinsamung und die Reduktion von körperlicher Aktivität unterstützt.

- Wichtig ist es, in diesen Kreislauf möglichst effektiv einzugreifen und im Verlauf des Lebens rechtzeitig Belastungen und Störungen der BBFunktion zu behandeln.

- Muskeltraining wird bereits als Standardbehandlung eingesetzt.

- Fasziale Techniken, die auf die Qualität und Funktion des Bindegewebes einwirken, würden sich als Behandlungsansatz für den BB anbieten.

- Diese Behandlungsansätze müssten standardisiert und auf ihre Wirksamkeit hin untersucht werden.

\section{Korrespondenzadresse

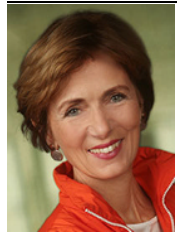 \\ Barbara Gödl-Purrer, MSc Physiotherapy Physiotherapie, FH JOANNEUM Eggenberger Allee 13, 8020 Graz, Österreich barbara@goedl-purrer.at}

Funding. Open access funding provided by FH Joanneum - University of Applied Sciences.

\section{Einhaltung ethischer Richtlinien}

Interessenkonflikt. B. Gödl-Purrer gibt an, dass kein Interessenkonflikt besteht.

Für diesen Beitrag wurden von den Autoren keine Studien an Menschen oder Tieren durchgeführt. Für die aufgeführten Studien gelten die jeweils dort angegebenen ethischen Richtlinien.

Open Access. Dieser Artikel wird unter der Creative Commons Namensnennung 4.0 International Lizenz veröffentlicht, welche die Nutzung, Vervielfältigung, Bearbeitung, Verbreitung und Wiedergabe in jeglichem Medium und Format erlaubt, sofern Sie den/die ursprünglichen Autor(en) und die Quelle ordnungsgemäß nennen, einen Link zur Creative Commons Lizenz beifügen und angeben, ob Änderungen vorgenommen wurden.

Die in diesem Artikel enthaltenen Bilder und sonstiges Drittmaterial unterliegen ebenfalls der genannten Creative Commons Lizenz, sofern sich aus der Abbildungslegende nichts anderes ergibt. Sofern das betreffende Material nicht unter der genannten Creative Commons Lizenz steht und die betreffende Handlung nicht nach gesetzlichen Vorschriften erlaubt ist, ist für die oben aufgeführten Weiterverwendungen des Materials die Einwilligung des jeweiligen Rechteinhabers einzuholen.

Weitere Details zur Lizenz entnehmen Sie bitte der Lizenzinformation auf http://creativecommons.org/ licenses/by/4.0/deed.de.

\section{Literatur}

1. Fritsch H (2015) Anatomie des weiblichen Beckenbodens. In: Michel M, Thüroff J, Janetschek G, Wirth M (Hrsg) Die Urologie. Springer Reference Medizin. Springer, Berlin, Heidelberg

2. Sapsford RR, Hodges PW, Richardson CA, Cooper DH, Markwell SJ, Jull GA (2001) Coactivation of the abdominal and pelvic floor muscles during voluntary exercises. Neurourol Urodyn 20(1):31-42

3. Sapsford RR (2004) Rehabilitation of pelvic floor muscles utilizing trunk stabilization. Man Ther 9:3-1

4. Talasz H, Kofler M, Kalchschmid E et al (2010) Breathing with the pelvic floor? Correlation of pelvic floor muscle function and expiratory flows in healthy young nulliparous women. Int Urogynecol J21:475-481 
5. Pool-Goudzwaard A, Hoek van Dijke G, van Gurp M, Mulder P, Snijders Ch, Stoeckart R (2004) Contribution of pelvic floor muscles to stiffness of the pelvic ring. Clin Biomech 19(6):P564-P571

6. Moser H, Leitner M, Baeyens J et al (2018) Pelvic floor muscle activity during impact activities in continent and incontinent women: a systematic review. Int Urogynecol J 29:179-196

7. Slieker-ten Hove MC, Pool-Goudzwaard AL, Eijkemans MJ, Steegers-Theunissen RP, Burger CW, Vierhout ME (2009) Pelvic floor muscle function in a general female population in relation with age and parity and the relation between voluntary and involuntary contractions of the pelvic floor musculature.Int Urogynecol JPelvicFloorDysfunct 20(12):1497-1504

8. TrowbridgeER, Wei JT, FennerDE, Ashton-Miller JA DeLancey JOL (2007) Effects of aging on lower urinary tract and pelvic floor function in nulliparous women. Obstet Gynecol 109(3):715-720

9. Burnett LA, Cook M, Shah S, Wong MM, Kado DM, Alperin M (2020) Age-associated changes in the mechanical properties of human cadaveric pelvic floor muscles. JBiomech 98:109436

10. Schleip R, Gabbiani G, Wilke J, Naylor I, Hinz B, Zorn A, Jäger H, Breul R, Schreiner S, Klingler W (2019) Fascia is able to actively contract and May thereby influence musculoskeletal dynamics: a histochemical and mechanographic investigation. Front Physiol 10:336

11. Proske U, Gandevia SU (2012) The proprioceptive senses: their roles in signalling body shape, body position and movement, and muscle force. Physiol Rev 92:1651-1697

12. De Lancey JOL, KaneLow L, Miller JM, Patel DA, Tumbarello JA (2008) Graphic integration of causal factors of pelvic floor disorders: an integrated lifespan model. Am J Obstet Gynecol 199(6):610.e1-610.e5

13. NygaardI, BarberMD, BurgioKL, KentonK, MeikleS, Schaffer J, Spino C, Whitehead WE, Wu J, Brody DJ, Pelvic Floor Disorders Network (2008) Prevalence of symptomatic pelvicfloor disorders in US women. JAMA 300(11):1311-1316

14. MacLennan AH, Taylor AW, Wilson DH, Wilson D (2000) The prevalence of pelvic floor disorders and their relationship to gender, age, parity and mode of delivery. Int J Obstet Gynaecol 107:1460-1470

15. Dumoulin C, Hunter KF, Moore K, Bradley CS, Burgio $\mathrm{KL}$, Hagen S, Imamura M, Thakar R, Williams K, Chambers T (2016) Conservative management for female urinary incontinence and pelvicorgan prolapse review 2013:Summary of the 5th International Consultation on Incontinence. Neurourol Urodynam 35:15-20

16. Dumoulin C, Cacciari LP, Hay-Smith EJC (2018) Pelvic floor muscle training versus no treatment, or inactive control treatments, for urinary incontinence in women. Cochrane Database Syst Rev. https://doi.org/10.1002/14651858.CD005654. pub2

17. Hay-Smith EJC, Herderschee R, Dumoulin C, Herbison GP (2011) Comparisons of approaches to pelvic floor muscle training for urinary incontinence in women. Cochrane Database Syst Rev. https://doi.org/10.1002/14651858. CD009508

18. Anderson CA, Omar MI, Campbell SE, Hunter KF, Cody JD, Glazener CMA (2015) Conservative management for postprostatectomy urinary incontinence.Cochrane Database SystRev. https:// doi.org/10.1002/14651858.CD009508

19. Stafford RE, Mazzone S, Ashton-Miller JA, Constantinou C, Hodges PW (2014) Dynamics of male pelvic floor muscle contraction observed with transperineal ultrasound imaging differ between voluntary and evoked coughs. J Appl Physiol 116(8):953-960

20. Rocha F, Carvalho J, Jorge Natal R, Viana R (2018) Evaluation of the pelvic floor muscles training in older women with urinary incontinence: a systematic review. Porto Biomed J3(2):e9

21. Sherburn M, Bird M, Carey M, Bø K, Galea MP (2011) Incontinence improves in older women after intensive pelvic floor muscle training: an assessor blinded randomized controlled trial. Neurourol Urodyn 30:317-324

22. Talasz $\mathrm{H}$, Gosch $\mathrm{M}$, Enzelsberger $\mathrm{H}$, Rhomberg HP (2005) Geriatrische Patientinnen mit Harninkontinenz-Symptomen und ihre Kontrolle über den Beckenboden. Z Gerontol Geriatr 38:424-430

23. Miller JM, Sampselle C, Ashton-Miller J et a (2008) Clarification and confirmation of the Knack maneuver: the effect of volitional pelvic floor muscle contraction to preempt expected stress incontinence. Int Urogynecol J 19:773-782. https://doi.org/10.1007/s00192-007-0525-3

24. Schleip R (2003) Fascial plasticity: a new neurobiological explanation. J Bodyw Mov Ther 7(1):11-19

25. Sammer A, Lackner R (2016) Der Beckenboden. Man Med 54:376-380. https://doi.org/10.1007/ s00337-016-0187-7

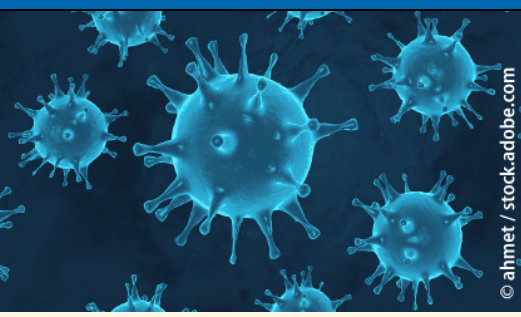

\section{Aktuelle Entwicklung zu COVID-19 bei Springer Nature und Springer Medizin}

Springer Nature und Springer Medizin unterstützen die globale Reaktion auf die COVID-19-Pandemie, indem ein schneller und direkter $\mathrm{Zu}$ gang zu den neuesten verfügbaren Forschungsergebnissen und Daten ermöglicht wird.

Auf der Homepage SpringerMedizin.de finden Sie ein immer aktuelles Dossier mit Beiträgen, Forschungsarbeiten und Ergebnissen zu SARS-CoV-2 sowie relevanten Links.

Darin z.B. auch die kürzlich publizierte

Empfehlung von DIVI, DGIIN, DGAI und DGP zur Intensivtherapie von Patienten mit COVID-19.

Springer Nature arbeitet mit globalen Organisationen zusammen, und verlinkt über SpringerNature.com/de auf eine eigene Landingpage mit einer Vielzahl an Information sowie freiem Zugriff auf die COVID-19-Contentplattformen von Nature Research, BioMed Central (BMC) und Springer.

Das Dossier zu Coronavirus / Covid-19 von Springer Medizin finden Sie hier: www.springermedizin.de/covid-19

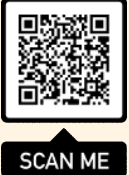

